\title{
Rehabilitation of a Patient with Temporomandibular Joint Disorder and Misocclusion Requiring Full Mouth Reconstruction, Based on the Multidisciplinary Approach: A Clinical Case
}

\author{
Fatima Dzalaeva ${ }^{1}$ Sergey Chikunov ${ }^{1,2}$ Anatoly Utyuzh ${ }^{1} \quad$ Maria Mikhailova ${ }^{1}$ Marzhanat Budunova ${ }^{1}$ \\ ${ }^{1}$ Department of Prosthodontics, First Moscow State Medical \\ University, Moscow, Russia \\ ${ }^{2}$ Department of Prosthodontics, Peoples Friendship University \\ (RUDN), Moscow, Russia \\ Address for correspondence Fatima Dzalaeva, PhD, Department \\ of Prosthodontics, First Moscow State Medical University, Bolshaya \\ Pirogovskaya Street, 19c1, Moscow 119146, Russia \\ (e-mail: dzalaevafati@rambler.ru).
}

Eur J Dent 2021;15:401-407

\begin{abstract}
Keywords

- edentulism

- dental prosthodontic rehabilitation

- temporomandibular joint

- condylography

- splint therapy

This case report involved the development of a rehabilitation system for patient with edentulism and an algorithm for diagnostic and therapeutic measures to be planned based on the patient's individual anatomical and physiological features. The results of treatment and rehabilitation made it possible to achieve the optimal distribution of loads on the dentition. It was shown that it is necessary to analyze casts in an articulator to reproduce a three-dimensional model of mandibular movements taking into account the individual features of the patient to obtain a satisfactory assessment and treatment planning of patients with edentulism requiring full mouth reconstruction. This case confirms that the developed algorithm is anatomically and pathogenetically justified, as it considers all changes and interrelationships of structures of the dentition.
\end{abstract}

\section{Introduction}

According to statistics, the number of people in Russia in need of dentures due to the absence of teeth is steadily increasing. ${ }^{1,2}$ A significant proportion of patients have a temporomandibular joint (TMJ) dysfunction, there is an asymmetry in the location of the joint condyle, accompanying by a change in the functioning of the dentition as a whole, which in turn cannot be ignored when using various prosthetic constructions. ${ }^{3-7}$

The complexity of the anatomical structure and biomechanics of this joint determines the high incidence of TMJ dysfunction, the pathology of which often acts as a trigger or supporting factor in the development of other diseases. .3,8-10 $^{2}$

The range of issues related to the diagnosis and treatment of TMJ pathology is an interdisciplinary problem. ${ }^{5,11-14}$ TMJ pathology is frequently observed in dental practice, while patients often complain of pain in the muscles of the

DOI https://doi.org/ 10.1055/s-0040-1717158 ISSN 1305-7456. maxillofacial area and arthralgia. This pain may persist for a long time, becoming very excruciating for patients. ${ }^{7,10,12,15}$ The pathogenesis of the previously mentioned disorders is inextricably linked with the state of occlusion. Due to this, it is important to detect disorders in the jaw relation; in particular, changes in the vertical occlusion, displacement of the mandible, etc., require the measurement of the centric relation.5.,16,17

As the main reasons for the development of disorders of the muscular-articular complex of the TMJ, two groups of factors are considered-occlusal articulating and psychogenic. Dysfunction of the TMJ has a multifactorial genesis, while the vast majority of TMJ pathologies are functionally determined. The etiology of TMJ dysfunction is caused by neuromuscular and occlusal-articulatory disorders. Neuromuscular syndrome is characterized by severe neuralgic pains of arthrogenic origin, myalgia, clicks in the joint, deviations with the phenomena of glossalgia, glossodynia, and bruxism..$^{14,17,18}$

(C) 2021. European Journal of Dentistry.

This is an open access article published by Thieme under the terms of the Creative Commons Attribution-NonDerivative-NonCommercial-License, permitting copying and reproduction so long as the original work is given appropriate credit. Contents may not be used for commercial purposes, or adapted, remixed, transformed or built upon. (https://creativecommons.org/licenses/by-nc-nd/4.0/) Thieme Medical and Scientific Publishers Pvt. Ltd., A-12, 2nd Floor, Sector 2, Noida-201301 UP, India 
The most important role in the development of TMJ diseases is played by malocclusion, which accounts for components ranging from 34 to $87 \%$ in the structure of this pathology. Most authors believe that the development of anomalies in the ratio of teeth is caused to a lesser extent by the morphological features of the elements of the joint and to a greater extent by changes in intra-articular relationships. Occlusion-related changes in the articular surfaces and TMJ capsules, as a rule, exacerbate age-related changes, which in turn lead to the emergence of anatomical prerequisites for the development of its dysfunction. ${ }^{19-21}$

The multifactoriality of the above problem largely determines the structure of the necessary diagnostic and therapeutic measures when planning orthopaedic rehabilitation. As part of a comprehensive interdisciplinary approach to diagnosis, it is necessary to use diagnostic research methods with the appropriate evidence base to verify the predominance of neuromuscular or occlusal-articular syndromes, as well as assess their systemic effect on the biomechanics of the musculoskeletal system. $5,7,22$

Currently, several conservative methods for correcting joint disorders have been proposed. In clinical practice, selective grinding, acupuncture, therapeutic exercises, autogenic training, and physiotherapeutic procedures are actively used. ${ }^{9,18,19}$ Together with other specialists, patients with TMJ dysfunction are prescribed drug therapy. Most often, such patients are prescribed sedatives, antidepressants, muscle relaxants, and antidepressants. Using this approach in patients with TMJ dysfunction determines the need to use a set of functional and radiation diagnostic methods, which allow obtaining a quantitative and qualitative analysis of the systems involved in the pathological process, and developing a targeted systemic treatment, rehabilitation program with subsequent quantitative and qualitative analysis of the effectiveness of this program..$^{1,4,5}$

The prosthodontic dentist should be focused on these aspects, and practical health care professionals should have an idea of the methods for examining patients with edentulism against the background of TMJ pathology and occlusion disorders requiring full mouth reconstruction. Several authors rightly believe that when examining such patients, the case history and use of the spectrum of necessary diagnostic methods are of great and sometimes crucial importance for establishing an accurate diagnosis and relevant planning of treatment and rehabilitation. ${ }^{15}$ In view of the above, it seems relevant to develop and improve approaches to dental prosthodontic treatment based on the use of an interdisciplinary approach and comprehensive diagnostics for a comprehensive assessment of the clinical presentation, as well as the development of a treatment plan taking into account the individual features of the patient.

The objective of the study is to increase the effectiveness of rehabilitation measures performed for patients in need of full mouth reconstruction.

\section{Case Report}

This case report presents a single clinical case with the results of the examination and treatment of patient $B$, age 43 years. The patient came to the clinic with complaints of muscle pain, pain during chewing and in masticatory muscles, as well as dissatisfaction with the aesthetic characteristics of the appearance. In the process of treatment and examination of patients requiring full mouth reconstruction in the facilities of Meditsina JSC Clinic, we have proposed a rehabilitation system based on an interdisciplinary approach. As part of the implementation of this approach when planning occlusion correction, the results of a physical examination of the patient that are obtained using a complex of diagnostic methods are considered.

When planning treatment, the anatomical and functional characteristics of the patient's dentition are studied with particular attention being paid to assessing the function of the TMJ and the presence of pathological signs of muscles disorders in the maxillofacial area.

The results of the aesthetic, clinical functional and instrumental analysis performed during diagnosis using the methods of condylography and cephalometry make it possible to determine the centric relation with casts mounted in the articulator. At the same time, an algorithm is used to work with the Gamma Dental program providing for digital occlusion simulation. The approach used provides for planning and correcting the occlusion, taking into account and timely correcting functional and aesthetic disorders in patients undergoing full mouth reconstruction.

Wax-up and manufacturing of dentures are performed using individual Gamma articulators, the advantages of which are cast mounting on an individual hinge axis, measuring the occlusive plane, and evaluating the gamma rotation. Wax-up with sequential opening performed in this way provides for obtaining high functional and aesthetic results of prosthodontic rehabilitation of patients requiring full mouth reconstruction. At the same time, an optimal distribution of loads on the dentition is achieved, the risk of veneering material chipping is reduced, and the hygiene of the oral cavity is also improved.

To diagnose the state of the dentition, methods of orthopantomography and condylography were used.

\section{Diagnostic}

The examination revealed difficulty in mastication, pain when opening and closing the mouth and in the TMJs, spasms in the neck and larynx, headaches, and postural disorders.

Palpation of the muscles of the maxillofacial area revealed pain in the posture muscles, muscle closers, openers-protractors, retractors, medial and lateral tractors, as well as changes in the position of the hyoid bone indicating swallowing problems.

The presence of muscle pain during chewing was an indication for further functional instrumental studies.

The view before treatment is presented in -Fig. 1. The analysis of casts of the maxilla and the mandible showed a mismatch between the active and passive central arches.

The results of the examination indicated that there were indications for expanding the scope of diagnostics and implementing a comprehensive functional instrumental study. An objective examination revealed the presence of the following 


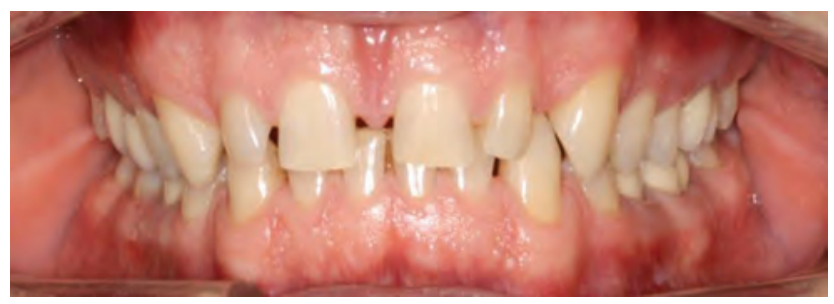

Fig. 1 Patient B. Intraoral photograph before treatment.

features: a deep incisal bite, diastemata, a decrease in the interincisal angle, a mismatch in the midlines, and dental class I.

Sagittal and transverse mismatch of the upper and lower dental arches was noted, cavities in teeth 17 and 27 , and root canal disorders in 16 and 26 were revealed on the enlarged $\mathrm{X}$-ray images.

In addition, speech disorders and disturbances of aesthetic characteristics of the maxillofacial area were identified.

The following treatment goals were formulated:

- Extraction of teeth 18 and 28

- Increase of the vertical occlusion, change in the depth of the occlusion

- Reshaping both upper and lower arches

- Changing the inclination of the occlusive plane (OPI)

- Changing the degree of the opening angle (DOA).

The following treatment plan is proposed:

- Condylography

- Professional oral cleaning

- Wax casting

- Root canal treatment

- Periotreatment

- Prosthodontic restoration.

In the process, individual wax casting parameters were determined using the Weber template and $X Y Z$ coordinate system to determine the location of holding dental cusps in the Cartesian coordinate system in the articulator space.

An important stage of the treatment was splint therapy; the treatment results were checked in a follow-up examination after 14 days.

According to the results of condylography, a decrease in the length of movement on the right, an increase in the activity of retractor muscles, and a mismatch of excursion and incursion movements were revealed.

In the right joint, protrusion and zero Bennett movement were noted, indicating disk adhesion or the development of an obstacle avoidance mechanism. There was detrusion in the left joint at the right mediotrusion, but the Bennett angle was positive.

When assessing the mouth's capability of opening and closing, it is revealed that the beginning and end of the movement are not coincident, implying nonstable occlusion and divergence between protractor and retractor muscles (-Fig. 2 ).

The revealed redetrusion indicates that during bruxism the maxilla does not overlap protrusion due to sharp slope of palatine surface of upper incisals, and the mandible is moved to the retrusion position with downward shear.

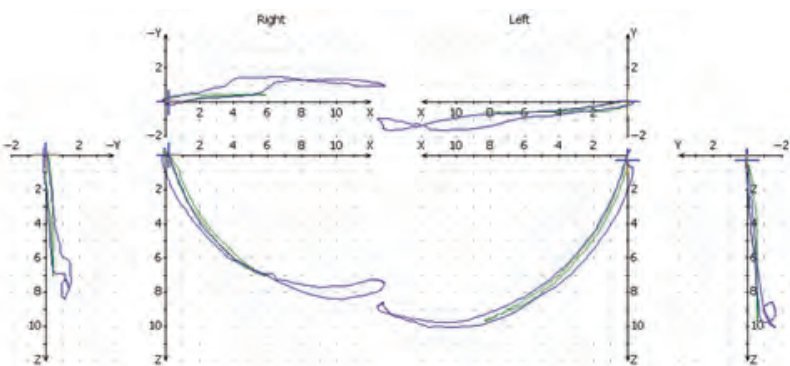

Fig. 2 Patient B condylography. Increased activity of retractor muscles. Mismatching of excursion and incursion movements.

There is overlay between speech movement and the first $4 \mathrm{~mm}$ of protrusion.

Gamma rotation indicates interference in the frontal area.

In general, the results of condylography show the increased activity of retractor muscles and mismatching of excursion and incursion movements.

Cephalometric analysis reveals the following characteristics:

- Increased occlusal vertical dimension

- Both jaws are in prognathic state, vertical occlusion can be increased from 45.7 to $47.5 \mathrm{~mm}$

- Incisal pin $+4.5 \mathrm{~mm}$

- OPI is decreased and it must be increased to 10 degrees for the right side and to 16 degrees for the left side

- Angle of the frontal restriction should be increased and OPI should be increased by 36 and 46 to obtain the opening angle of 10 degrees.

Articulator settings: The right sagittal condylar path (SCI) is 57 and the left one is 63, OPI (right) is 10 and left is $16, \mathrm{DOA}=17$.

Anterior guidance (AG) generates 75 degrees.

\section{Treatment}

Coordinates of mandibular cusps are determined using Weber template and added to the table for calculating the angle for each of the teeth. We remounted the casts after splint therapy for a wax-up (-Fig. 3).

Casts were remounted in the articulator in a new therapeutic position for diagnostic wax-up in 3 weeks after completing the spit therapy. Frontal restriction, canine guidance, and retrusion restriction on the first maxillary and mandibular molars are shown in -Fig. 4.

Consistent guidance was made on all buccal teeth, including the retrusion restriction. The final result of the treatment is presented in - Fig. $\mathbf{5}$.

In - Fig. 6, there is orthopantomographical image and intraoral photograph of the patient's teeth and gingiva in 9 years after the treatment.

\section{Discussion}

In the past decade, the emergence of new methods of restoration and replacement of lost teeth calls for a better understanding of the mechanism of dentofacial system, 

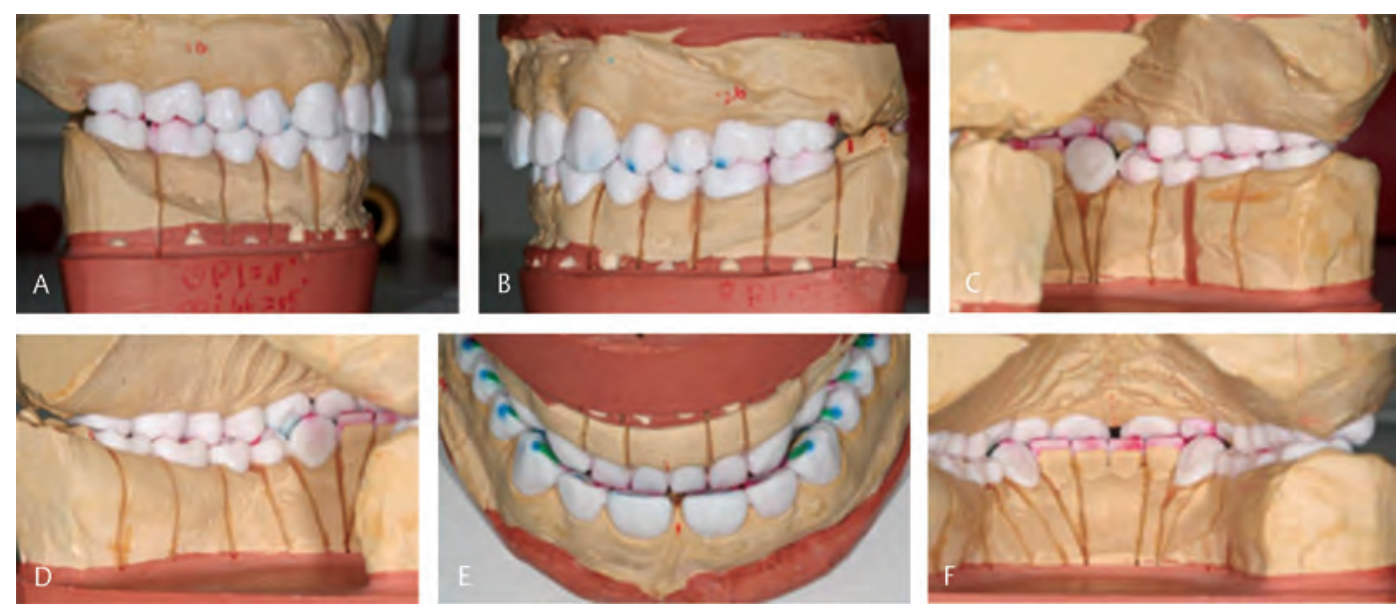

Fig. 3 Remounting of the casts after splint therapy for a wax-up. (A) Dental class I on the right; (B) dental class I on the left; (C) holding cusps of the maxilla in intercusp fissure. Mandibular molars. Right-side view, (D) holding cusps of the maxilla in intercusp fissure. Mandibular molars. Left-side view, (E) overbite depth and width created taking into account the function of speech and frontal restriction; (F) canine guidance and frontal restriction.
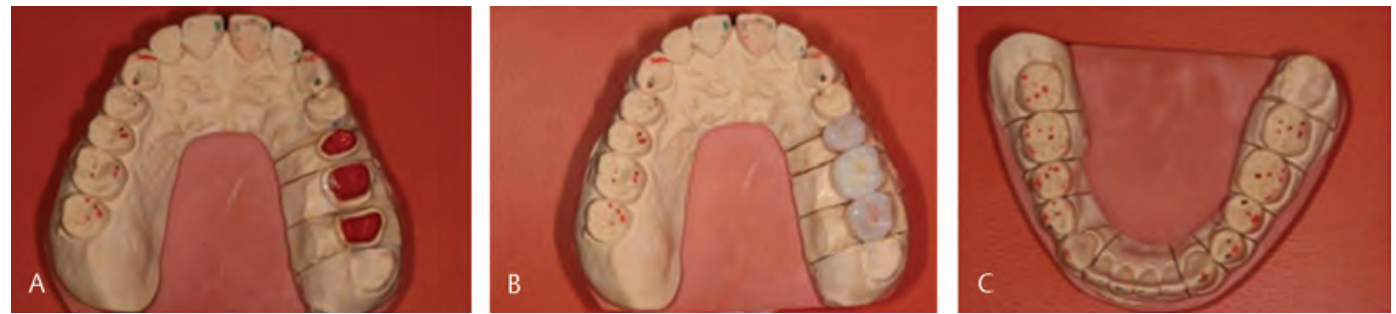

Fig. 4 Reconstruction of frontal restriction, canine guidance, and retrusion restriction on the first maxillary and mandibular molars. (A) Paths of frontal and canine guidance on the canines and maxillary incisors; (B) manufacture of all-ceramic restoration on teeth 25, 26, and 27; (C) three-pointed contact in maxillary and mandibular cusps and intercusp fissures of the mandibular molars.
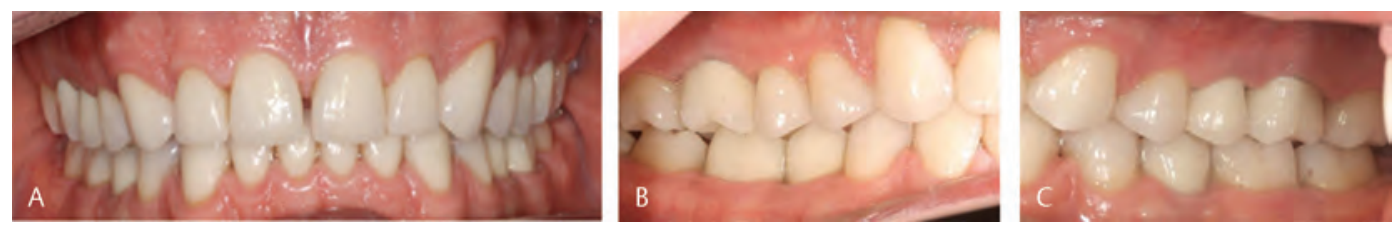

Fig. 5 Patient B. The final result of the treatment. (A) Frontal plane; (B) occlusal plane of the maxillary teeth; and (C) occlusal plane of the mandibular teeth.
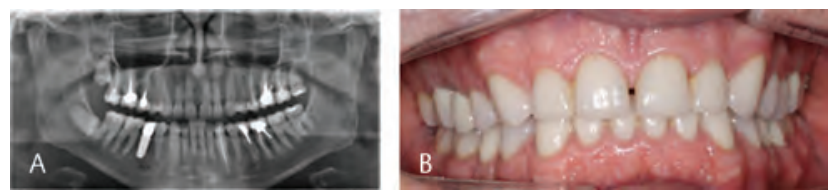

Fig. 6 Patient B. State of the oral cavity in 9 years after the treatment. (A) Orthopantomography and (B) intraoral photograph.

TMJ and occlusive disorders, and the influence of occlusion on the state of teeth and periodontium. Also, the assessment of all characteristics after the prosthetic restorations in the process of further patient observation is extremely important. ${ }^{2,4-7}$

TMJ is a biological microsystem that includes several subsystems (articular cavity, disc, and bilaminar zone) and plays the role of a shock-absorbing and stabilizing mechanism that ensures the position of mandibula capture with the glenoid fossa at different phases of jaw movement. Regenerative processes occurring in the fibrocartilaginous tissue of the disc and joint surfaces provide the congruence of the surfaces of these elements. ${ }^{5,11,14-16}$ Violations of these processes, observed in various diseases and injuries, can lead to deformations of articular structures, manifested by several clinical signs of TMJ pathology. 19,23,24

Nowadays, more and more researchers have come to realize that functional duration of prosthodontic structures mainly depends on properly created occlusion relations. ${ }^{13-16}$ Many researchers in their works consider physiological occlusion as one of the stages of rehabilitation treatment. Dental occlusion is traditionally considered as "the static relationship between the cutting and chewing surfaces of the teeth (or their prostheses) of the upper and lower jaws."25 Currently, most authors believe that occlusion should be analyzed in 
three spatial dimensions, given that its maintenance is provided by the anatomical relationships and functional characteristics of the teeth, chewing and facial muscles. ${ }^{12-14}$

It is believed that occlusion disorders are based on:

- Changes in the muscles of the maxillofacial region, in particular, compensatory muscle contractions with the subsequent development of pain syndrome. In this case, the correct position of the caput mandibulae can be temporarily maintained, the articular disc does not move.

- Long-term occlusal relationships violations by the type of compression cause adaptive changes in tissues-muscle, connective, and bone, as a result of which there are morphological changes in the articular disc, surfaces, ligaments, and the bilaminar zone. Subsequently, disc dislocation and changes in the tone of the masticatory muscles occur., ${ }^{5,20}$

The most common of dentoalveolar anomalies is distal occlusion of the dentition, the frequency of its occurrence in the adult population is, according to various sources, from 13 to $57 \% \%^{3,8}$ Violations of occlusion lead to an increase in the load on the TMJ, while the pressure on its constituent structures increases. ${ }^{17}$ Prolonged exposure with the development of degenerative changes in conditions of a decrease in the adaptive mechanisms of tissue structures leads to TMJ pathology, which is combined with impaired coordination of the function of the masticatory muscles and harmonious mandibula movements. ${ }^{4,9}$

Assessing the condition of the dentition, in general, and the TMJ, in particular, when examining a patient, one should take into account not only the history and clinical manifestations but also analyze the complex of anthropometric and functional parameters obtained using appropriate diagnostic methods. . $13,22^{2}$

Today, most authors consider occlusive disorders as potential factors in the development of functional disorders in the TMJ. ${ }^{12-15}$ Thus, when performing a set of measures for the correction and restoration of the anatomical shape of the teeth, one of the key areas should be the correction of the pathology of the TMJ. The most important stage in the implementation of our approach is a complete and consistent examination of patients in need of total restoration of the dentition. In this case, it is necessary to make efforts aimed at identifying the cause-and-effect relationships of pain sensations with occlusion disorders and TMJ pathology.

When the sufficient amount of occlusal contacts and the TMJ elements are properly located, the formation of physical occlusion is the base for balanced distribution of functional and parafunctional loads. ${ }^{5,14,17,18}$

Indeed, there is a need for detailed patient history, clinical extra- and intraoral examinations, and X-ray imaging before and during the prosthetic treatment process in which the occlusion is changed or restored. ${ }^{17,18}$

\section{Conclusion}

In view of the above and on the basis of the case described in the study, we provide ways of application of information on the compensation mechanism when creating dentures for patients in need of full mouth reconstruction. The applied approach makes it possible to replace the orthodontic treatment with the prosthodontic one, including the aesthetic parameters. In this case, in the process of diagnosis and treatment of the patient involved in the study, we have evaluated the possibility of creating a canine guidance and frontal and retrusion restrictions. The sagittal and transversal alignments of active and passive dental arches in the maxilla and mandible were performed. For this, we used the findings from condylographic studies, cephalometric analysis, and analysis of models, combined these findings with the results of muscle palpation, which were used in the process of wax casting. The individual wax casting parameters were determined using the Weber template using the XYZ coordinate system and were used to determine the location of the holding dental cusps in the Cartesian coordinate system in the articulator space.

According to our view, adequate evaluation and treatment planning require analysis of casts in the articulator to enable creating a three-dimensional model of the mandible movement toward the maxilla taking into account the patient's individual features.

The designed algorithm of dental prosthodontic rehabilitation for patients with edentulism in need of full mouth reconstruction is anatomically and pathogenetically justified, as the whole complex of changes of the dental system structures and their interactions with other body systems which underlie the clinical manifestations in this patient category was taken into account in the course of its implementation.

Timely prosthodontic treatment for eliminating occlusal disorders because of deformity of dentitions should include preventive measures against profound changes in the anatomical and functional nature of the dental system of patients with edentulism in need of full mouth reconstruction.

\section{Funding \\ None.}

\section{Conflict of Interest}

None declared.

\section{References}

1 Kamieva NA, Kausova GK, Ruzuddinov SR. On the need for dental restorations in elderly persons. J Kazakh Nat Med Univ 2018;4:41-45

2 Semenyuk VM, Akhmetov EM, Fedorov VE. Veyrune JL, Nicolas E, Marion Bessadet M. Results of arrangement and efficiency of prosthodontic treatment and quality of dentures (social research data) Inst Stomatol 2017;1(74):26-29

3 Furlan RM, Giovanardi RS, Britto AT, Oliveira e Britto DB. The use of superficial heat for treatment of temporomandibular disorders: an integrative review. CoDAS 2015;27(2):207-212

4 Saakian ShKh, Kalamkarov AE. The analysis of changes in bone at orthopedic treatment of patients with defects of tooth alignments with use the dental implants. Rus J Dentist 2014;2:13-16

5 Dolce MC, Aghazadeh-Sanai N, Mohammed S, Fulmer TT. Integrating oral health into the interdisciplinary health sciences curriculum. Dent Clin North Am 2014;58(4):829-843 
6 Chikunov So. A sparing method of dental restoriation without prior preparation of abutment teeth. Dental Institute 2012;2:52-53

7 Babich VV, Iordanishvili AK, Okunev MA, et al. Arrangement of Diagnostics and Treatment of Temporomandibular Joint Disorders, Taking into Account the Adaptational Capability of the Human Body St. Petersburg: Nordmedizdat; 2016

8 Ali DA. Patient satisfaction in dental healthcare centers. Eur J Dent 2016;10(3):309-314

9 Timoshin AV, Sevbitov AV, Drobot GV, Yumashev AV, Timoshina MD. Use of bioresorbable plates on the basis of collagen and digestase for treatment of diseases of oral mucosa (review of clinical cases) Int J Green Pharm 2018;12(S1): 290-296

$10 \mathrm{He} \mathrm{H}$, Liu ZJ. [Risk of temporomandibular joint and its management in orthodontic treatment]. Zhonghua Kou Qiang Yi Xue Za Zhi 2019;54(12):808-814

11 Uzuner FD, Aslan BI, Dinçer M. Dentoskeletal morphology in adults with Class I, Class II Division 1, or Class II Division 2 malocclusion with increased overbite. Am J Orthod Dentofacial Orthop 2019;156(2):248-256.e2

12 Utyuzh AS, Yumashev AV, Mikhailova MV. Spectrographic analysis of titanium alloys in prosthetic dentistry. J Glob Pharma Technol 2016;8(12):7-11

13 Fonseca EPD. Multivariate method to identify inequalities in oral healthcare access. Eur J Dent 2018;12(4):475-479

14 Trezubov VN, Chikunov SO, Bulycheva EA, et al. Incremental wax-up of complete denture in complex clinical presentation. Rinsho Shika 2017;3(83):60-63

15 Trezubov VN, Bulycheva EA, Chikunov SO, et al. Cephalometric examination of the facial skeleton when planning correction of misalignment of dental occlusion surfaces. Dent Inst 2015;4(69):102-104

16 Bakke M, Petersson A, Wiesel M, Svanholt P, Sonnesen L. Bony deviations revealed by cone beam computed tomography of the temporomandibular joint in subjects without ongoing pain. J Oral Facial Pain Headache 2014;28(4):331-337

17 Chikunov SO. Repeated rehabilitation of patients after prosthodontic dental treatment. St. Petersburg: Author's abstract of doctoral dissertation of Doctor of Medical Sciences; 2014

18 Chatzopoulos GS, Wolff LF. Symptoms of temporomandibular disorder, self-reported bruxism, and the risk of implant failure: A retrospective analysis. Cranio 2020;38(1):50-57

19 Saeidi Pour R, Engler MLPD, Edelhoff D, Prandtner O, Frei S, Liebermann A. A patient-calibrated individual wax-up as an essential tool for planning and creating a patient-oriented treatment concept for pathological tooth wear. Int J Esthet Dent 2018;13(4):476-492

20 Gil-Martínez A, Paris-Alemany A, López-de-Uralde-Villanueva I, La Touche R. Management of pain in patients with temporomandibular disorder (TMD): challenges and solutions. J Pain Res 2018;11:571-587

21 Yumashev AV, Utyuzh AS, Volchkova IR, Mikhailova MV, Kristal EA. The influence of mesodiencephalic modulation on the course of postoperative period and osseointegration quality in case of intraosseous dental implantation. Ind J Sci Techn 2016;9(42):104307-104307

22 McSwiney TP, Collins JM, Bassi GS, Khan S. The interdisciplinary management of hypodontia patients in the UK: a national service evaluation. Br Dent J 2017;222(1):31-35

23 Canut Brusola JA, Aparato estomatognático: diseño biomecánico. In: Canut Brusola JA, ed. Ortodoncia Clínica Y Terapéutica, 2nd ed. Barcelona: Masson S.A.; 2005 17-24

24 Paço M, Chaves P, Pinho F, et al. Common symptoms of temporomandibular disorders do not mean same treatment plans: a case series. Int Orthod 2018;16(1):174-214

25 Ferro KJ, Morgano SM, Driscoll CF, et al. The glossary of prosthodontic terms: ninth edition. J Prosthet Dent 2017;117(5S): e1-e105 